\title{
The Incidence of Different Candida Albicans Infections in Durres Area
}

\author{
PhD Cand. MSc. Anisa Liti ${ }^{1}$ \\ Dr. Alba Dako² \\ Prof. Dr. Margarita Hysko³ \\ ${ }^{1}$ Department of Medicine, Professional Studies Faculty, AleksandërMoisiu University, Durrës, Albania \\ 2 Responsible of Microbiology Laboratory of DRSH, Durrës, Albania \\ 3Department of Biology, Natural Sciences Faculty, Tirana University, Albania; Email'anisa.liti@yahoo.com
}

\section{Doi:10.5901/ajis.2015.v4n3s1p450}

\section{Abstract}

\begin{abstract}
Candida albicans is an opportunistic fungal pathogen that is responsible for candidiasis in human hosts. C. albicans grow in several different morphological forms, ranging from unicellular budding yeast to true hyphae with parallel-side wall. Its ability to transform from yeast to hyphae is considered as a virulence factor. The vast majority of Candida infections are mucosal, but in severe cases, the fungus can penetrate through epithelial layers into deeper tissues, reach the blood stream and, from there, may cause life-threatening systemic infections. Our study was carried out between July 2013 and June 2014. This study is part of a 3 -year study related with C. albicans infections in Durres area. In previous studies we have found the relation of these infections by gender, age groups, seasons. In this study we wanted to show which of these C. albicans infections was the most prevalent for this period of time in Durres area. A number of 2641 persons suspected for $C$. albicans intestinal, cutaneous, throat or genital infections, came in the Laboratory of Microbiology, DRSH, Durres, Albania, from July 2013 till June 2014. 655 persons out of 2641 were suspected for $C$. albicans feaceal infections, 97 persons out of 2641 were suspected for $C$. albicans cutaneous infections, 764 out of 2641 persons were suspected for $C$. albicans throat infections, 1125 out of 2641 persons were suspected for $\mathrm{C}$. albicans genital infections. It has resulted that the most prevalent $\mathrm{C}$. albicans infections in Durres area, for the period of time time July 2013 - June 2014 were the cuteneous infections.
\end{abstract}

Keywords: Candida albicans, candidiasis, hyphea, mucosal infections, systemic infections

\section{Introduction}

Candida albicans taxonomy: Domain - Eukaryota, Phylum - Ascomycota, Class - Saccharomycetes, Order Saccharomycetales, Family - Saccharomycetaceae, Genus - Candida, species= albicans (MicrobeWiki, 2014).

While almost all of us possess Candida albicans in our oral cavity, gastrointestinal tracts, genitourinary tracts, and on skin as a relatively harmless commensal organism, $C$. albicans is a major systemic fungal pathogen in humans (Gow $\mathrm{N}$ et. al., 2011). C. albicans is an opportunistic fungal pathogen that is responsible for candidiasis in human hosts. $C$. albicans grow in several different morphological forms, ranging from unicellular budding yeast to true hyphae with parallel-side wall (Sudbery P. et. al., 2004). Its ability to transform from yeast to hyphae is considered as a virulence factor. Hyphae formation from yeast cells is a virulence trait that enables the human opportunistic pathogen $\mathrm{C}$. albicans to invade host tissues (Farahnaz Bineshian et. al., 2015). Hyphal differentiation in C. albicans results from an integration of signaling events from environmental cues that can act either alone or in combination. These include elevated temperature, $\mathrm{pH}$ or $\mathrm{CO}_{2}$, limiting $\mathrm{O}_{2}$, nitrogen or carbon, and exposure to serum, GlcNAc and other less well defined inducers. Signaling to Ras1, adenylate cyclase (Cdc35/Cyr1) and the APSES transcription factor Efg1 provides a link that integrates many of these disparate environmental signals (Michael L. Pendra et. al., 2015).

The vast majority of Candida infections are mucosal, manifesting as vaginal or oral candidiasis, which together account for an estimated 40 million infections per year (Julian R. Naglik et. al., 2014). In severe cases, the fungus can penetrate through epithelial layers into deeper tissues, reach the blood stream and, from there, may cause lifethreatening systemic infections (Betty Wächtler et. al., 2011).

The interaction between $C$. albicans and the host during infection is a complex and dynamic process. $C$. albicans utilizes different strategies to cope with the host's environmental cues for morphological change, proliferation, and escape from the host's immune defenses (Yan Yu Chen et. al., 2013). An important attribute of microbial adhesive proteins is the 
presence of determinants that confer targeting to the yeast or hyphal cell surface. Als1p and Ala1p, encoded by members of the large ALS gene family were reported to adhere to endothelial and epithelial cells, and extracellular matrix proteins (Paula Sundstrom 2002).

In the routine diagnostic microbiology laboratory, C. albicans can be identified presumptively with simple, rapid, and inexpensive methods such as germ tube or colorimetric tests, as well as the use of selective chromogenic agar media (Jan Trnovsky et. al., 2008).

\section{Materials and Methods}

This study was carried out between July 2013 and June 2014. A collection of 2641 yeast strains was obtained by routine isolation from feacal, cutaneous, oral, spermatic and vaginal swabs in the Laboratory of Microbiology, DRSH, Durres, Albania.

All specimens have had a label completely filled with the patient's name, age, sex and specimen source. Swabs were immediately transferred to Sabouraud's Dextrose Agar (SDA). Than culture tubes were incubated at $30^{\circ} \mathrm{C}$ for $24-48$ hours.

For C. albicans identification we used Microscopy Identification. The microscope is the best tool for a microbiologist. Distinct features of yeasts can be identified by observing their morphology. Microscopes can be used for fast identification and detection of possible yeasts in a clinical sample. When C. albicans was present, short, slender, tubelike structures (germ tube) were observed under the microscope.

\section{Results and Discussions}

This study is part of a 3 -year study related with $C$. albicans infections in Durres area. In previous studies we have found the relation of these infections by gender, age groups, seasons. In this study we wanted to show which of these $C$. albicans infections was the most prevalent for this period of time in Durres area.

In this study we have analysed 2641 individuals suspected for some different $C$. albicans infections. The samples taken from these persons were obtained by routine isolation from feacal, cutaneous, oral, spermatic and vaginal swabs in the Laboratory of Microbiology, DRSH, Durres, Albania. The samples were taken from July 2013 till June 2014.

655 persons out of 2641 were suspected for $C$. albicans feaceal infections, 97 persons out of 2641 were suspected for $C$. albicans cutaneous infections, 764 out of 2641 persons were suspected for $C$. albicans throat infections, 1125 out of 2641 persons were suspected for $C$. albicans genital infections.

The chart below shows the percentage of the persons analyzed for each C. albicans infections.

Chart 1. The percentage of the persons analyzed for each C. albicans infections

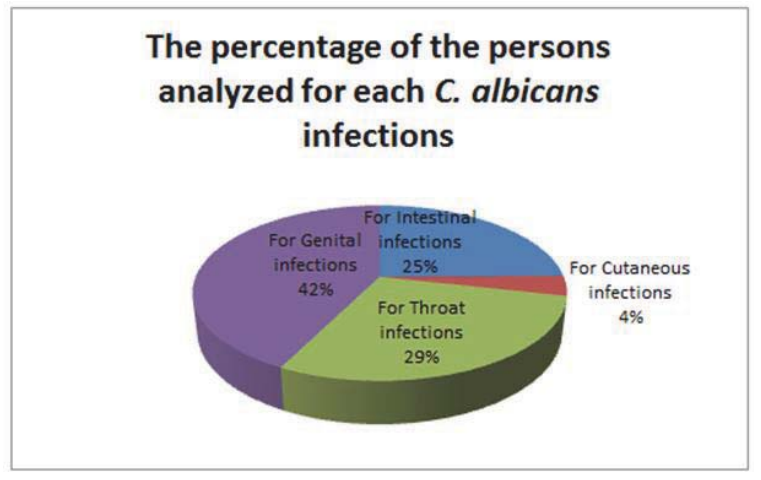

To analyze C. albicans intestinal infections, we have collected 655 samples from persons suspected, 446 of which resulted negative and 209 resulted positive. The number of positive and negative $C$. albicans intestinal infections is shown in the chart below. 
Chart 2. The number of negative and positive $C$. albicans intestinal infections

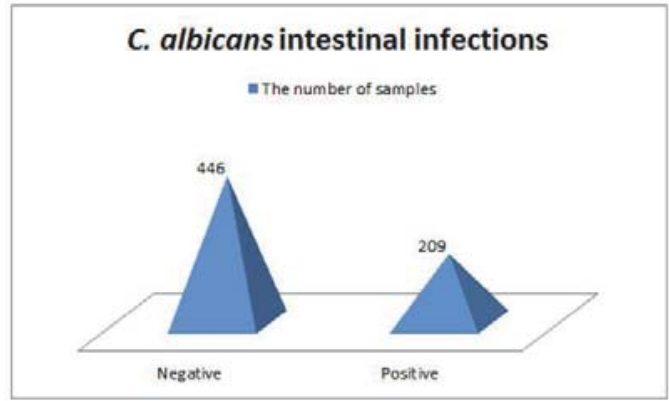

A collection of 97 cutaneous swabs form persons suspected for $C$. albicans cutaneous infections, was obtained to analyze the incidence of these infections. 32 out of 97 samples resulted negative, 65 out of 97 samples resulted positive. The number of positive and negative $C$. albicans cutaneous infections is shown in the chart below.

Chart 3. The number of negative and positive $C$. albicans cutaneous infections

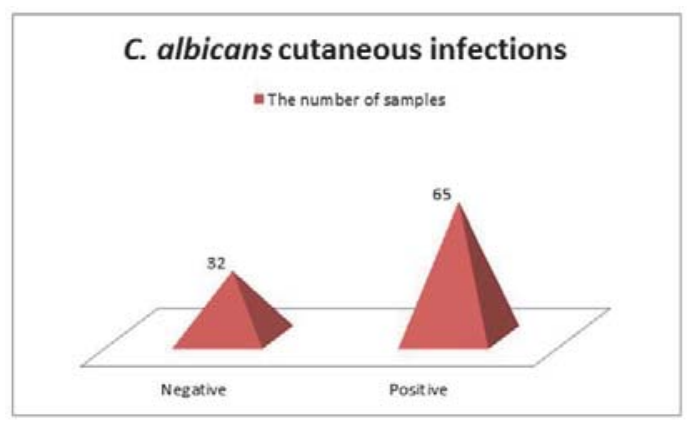

According to $C$. albicans throat infections, we have collected a total number of 764 samples. The analyzes showed that 573 out of 764 samples resulted negative and 191 out of 764 samples resulted positive. This incidence of $C$. albicans throat infections is shown in the chart below.

Chart 4. The incidence of of $C$. albicans throat infections

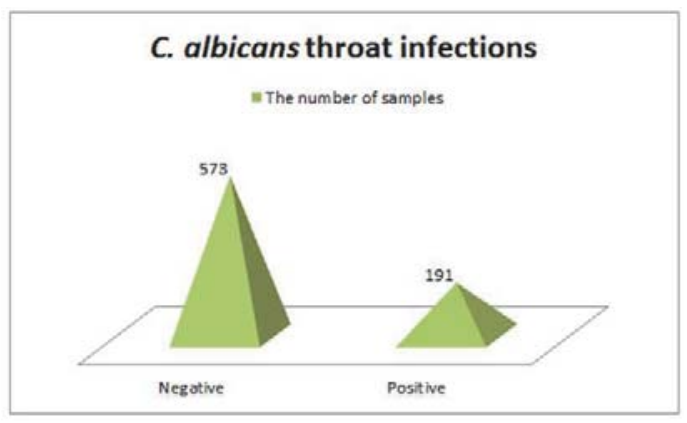

To analyze C. albicans genital infections, we have collected 1125 samples, 890 of which resulted negative and 235 resulted positive. The number of positive and negative $C$. albicans genital infections is shown in the chart below. 
Chart 5. The number of negative and positive $C$. albicans genital infections

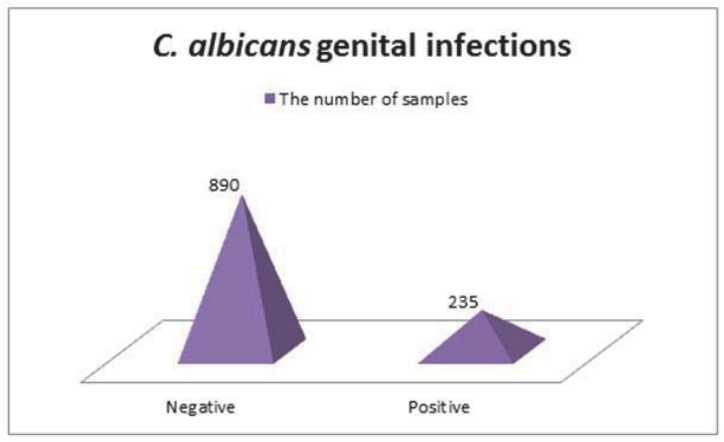

In this study we wanted to analyze the incidence of $C$. albicans infections, which of them are more prevalent in Durres area for this period of time. The processing of the data showed that only $32 \%$ of persons suspected for $C$. albicans intestinal infections resulted positive; $67 \%$ of persons suspected for $C$. albicans cutaneous infections resulted positive; only $25 \%$ of persons suspected for $C$. albicans throat infections resulted positive; only $21 \%$ of persons suspected for $C$. albicans genital infections resulted positive. The chart below shows the incidence of $C$. albicans infections for the period of time July 2013 - June 2014, in Durres area.

Chart 6. The incidence $C$. albicans infections

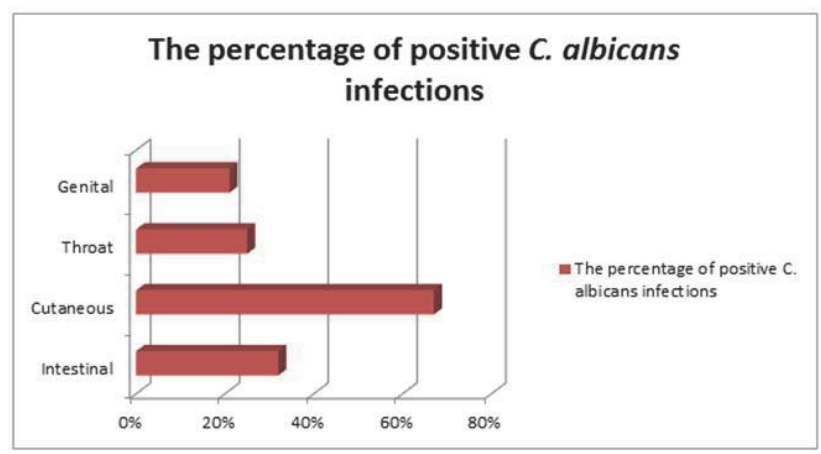

As shown in the chart above, the most prevalent $C$. albicans infections in Durres area, for the period of time time July 2013 - June 2014 are the cutaneous infections. Which are followed by intestinal infections and throat infections. The lowest number of $C$. albicans infections is occupied by $C$. albicans genital infections. The number of persons analyzed for C. albicans cutaneous infections is smaller than the others, but the percentage of positive cases resulted much higher. So we can say that may be clinical signs appeared in skin during C. albicans cutaneous infections are more specific, compared with $C$. albicans intestinal, throat and genital infections, whose symptoms can be confused with other microorganism infections.

\section{References}

Betty Wächtler, Duncan Wilson, Katja Haedicke, Frederic Dalle, Bernhard Hube (2011) From Attachment to Damage: Defined Genes of Candida albicans Mediate Adhesion, Invasion and Damage during Interaction with Oral Epithelial Cells. Plos collections DOI: 10.1371/journal.pone.0017046

Farahnaz Bineshian, Mohammad Hossien Yadegari, Zohre Sharifi, Mohammadreza Akbari Eidgahi, Reza Nasr (2015) Identification of Candida Species Using MP65 Gene and Evaluation of the Candida albicans MP65 Gene Expression in BALB/C Mice. J Microbiol doi: 10.5812/jjm.8(5)2015.18984 PMCID: PMC4458353 
Gow N, van de Veerdonk FL, Brown AJ, Netea MG. (2011) Candida albicans morphogenesis and host defence: discriminating invasion from colonization. Nat Rev Microbiol. 12;10(2):112-22. doi: 10.1038/nrmicro2711.

Jan Trnovsky, William Merz, Phyllis Della-Latta, Fann Wu, Maiken Cavling Arendrup and Henrik Stender (2008) Rapid and Accurate Identification of Candida albicans Isolates by Use of PNA FISH. J. Clin. Microbiol. vol. 46 no. 4 1537-1540 doi: 10.1128/JCM. 00030-08

Julian R. Naglik , Jonathan P. Richardson, David L. Moyes (2014) Candida albicans Pathogenicity and Epithelial Immunity. Plos Collections DOI: 10.1371/journal.ppat.1004257

Michael L. Pendra, David D. Robert, Scott G. Filler (2015) Hbr1 Activates and Represses Hyphal Growth in Candida albicans and Regulates Fungal Morphogenesis under Embedded Conditions. PLoS One; 10(6): e0126919. doi: 10.1371/journal.pone.01269 19PMCID: PMC4454550

Paula Sundstrom (2002) Adhesion in Candida spp. Cellular Microbiology Volume 4, Issue 8, DOI: 10.1046/j.1462-5822.2002.00206.x pages 461-469

Sudbery P, Gow N, Berman J. ( 2004) The distinct morphogenic states of Candida albicans. Trends in Microbiology 12(7):317-24.

The student-edited microbiology resource Candida albicans (2014), MicrobeWiki

Yan Yu Chen, Chun-Cheih Chao, Fu-Chen Liu, Po-Chen Hsu, Hsueh-Fen Chen, Shih-Chi Peng, Yung-Jen Chuang, Chung-Yu Lan, Wen-Ping Hsieh, David Shan Hill Wong (2013) Dynamic Transcript Profiling of Candida albicans Infection in Zebrafish: A Pathogen-Host Interaction Study. Plos collections DOI: 10.1371/journal.pone.0072483 